\title{
Impact of MR on mature adipocytes in high-fat/high-sucrose diet-induced obesity
}

\author{
Tomoaki Hayakawa', Tomomi Minemura', Toshiharu Onodera', Jihoon Shin',2, Yosuke Okuno', Atsunori Fukuhara1,3, \\ Michio Otsuki' and lichiro Shimomura'
}

1Department of Metabolic Medicine, Osaka University Graduate School of Medicine, Osaka, Japan

2Department of Diabetes Care Medicine, Osaka University Graduate School of Medicine, Osaka, Japan

${ }^{3}$ Department of Adipose Management, Osaka University Graduate School of Medicine, Osaka, Japan

Correspondence should be addressed to A Fukuhara: fukuhara@endmet.med.osaka-u.ac.jp

\section{Abstract}

Active glucocorticoid levels are elevated in the adipose tissue of obesity due to the enzyme 11 beta-hydroxysteroid dehydrogenase type 1. Glucocorticoids can bind and activate both glucocorticoid receptor (GR) and mineralocorticoid receptor (MR), and pharmacological blockades of MR prevent high-fat diet-induced obesity and glucose intolerance. To determine the significance of MR in adipocytes, we generated adipocytespecific MR-knockout mice (AdipoMR-KO) and fed them high-fat/high-sucrose diet. We found that adipocyte-specific deletion of MR did not affect the body weight, fat weight, glucose tolerance or insulin sensitivity. While liver weight was slightly reduced in AdipoMR-KO, there were no significant differences in the mRNA expression levels of genes associated with lipogenesis, lipolysis, adipocytokines and oxidative stress in adipose tissues between the control and AdipoMR-KO mice. The results indicated that MR in mature adipocytes plays a minor role in the regulation of insulin resistance and inflammation in high-fat/high-sucrose diet-induced obese mice.

\author{
Key Words \\ - mineralocorticoid \\ receptor \\ - adipocyte \\ - obesity \\ - insulin resistance
}

\section{Introduction}

The mineralocorticoid receptor (MR) is a member of the steroid receptor family of ligand activated transcription factors. MR is expressed in several tissues, including the kidney, heart, colon, brain and adipose tissue (Armani et al. 2015). In the kidney, its activation leads to the expression of $\mathrm{ENaC}$ or $\mathrm{Na} / \mathrm{K}$ ATPase resulting in the reabsorption of sodium to maintain a normal salt concentration in the body. Consequently, systemic MR-deficient mice die from dehydration approximately 10 days after birth (Berger et al. 1998). MR binds both aldosterone and glucocorticoids (Reul \& de Kloet 1985), and circulating levels of endogenous glucocorticoids are 100-1000 times higher than those of aldosterone. In epithelial tissues, 11 beta-hydroxysteroid dehydrogenase type 2 (11bHSD2) inactivates glucocorticoids, resulting in selective activation of MR by aldosterone (Funder 2009). On the contrary, in nonepithelial tissues lacking 11bHSD2, such as the adipose tissue, MR is activated by glucocorticoids due to their higher circulating concentrations than aldosterone (Funder 2000, Caprio et al. 2007).

In obesity, circulating glucocorticoid levels are normal; however, active glucocorticoid levels are elevated in the adipose tissue due to the enzyme 11 beta-hydroxysteroid dehydrogenase type 1 (11bHSD1) (Masuzaki et al. 2001; Engeli et al. 2004; Patsouris et al. 2009). Furthermore, MR is overexpressed in the adipose tissue of a mouse model of metabolic syndrome and also in obese individuals (Hirata et al. 2009, 2012; Urbanet et al. 2015). 
Treatment with eplerenone, a specific MR blocker, partially reverses the obesity-associated dysfunction of adipocytes and insulin resistance in mice (Guo et al. 2008; Hirata et al. 2009). Moreover, transgenic mice with adipocyte-specific inducible overexpression of MR are overweight with large fat mass, insulin resistance and features of metabolic syndrome (Urbanet et al. 2015). Thus, MR seems a suitable target molecule for locally activated glucocorticoid in the adipose tissues of obesity. However, no information is available on adipocyte-specific MR-deficient mice. In this study, we describe the generation of adipocytespecific MR-deficient (AdipoMR-KO) mice and report about the contribution of MR in obesity-related metabolic syndrome.

\section{Materials and methods}

\section{Animal experiments}

All animal studies were approved by the Ethics Review Committee for Animal Experimentation of Osaka University, Graduate School of Medicine and performed in accordance with the Osaka University Institutional Animal Care and Use Committee Guidelines. To establish the AdipoMR-KO mice, we crossed MR-floxed (McCurley et al. 2012) and Adiponectin-Cre (Eguchi et al. 2011) mice. The MR-floxed mice were kindly provided by Prof. Pierre Chambon (Institute for Genetics and Cellular and Molecular Biology, Strasbourg, France) and Prof. Iris Z Jaffe (Tufts University, Medford, MA, USA) and Adiponectin-Cre mice by from Drs E Rosen (Beth Israel Deaconess Medical Center, Boston, MA, USA) and J Eguchi (Okayama University, Okayama, Japan). Mice were fed a normal chow diet (protein, 25.7\%; lipid, $12.8 \%$; carbohydrate, $61.6 \%$ by calories; Oriental Yeast, Tokyo, Japan) or a high-fat/high-sucrose diet (HFHSD; protein, 17.2\%; lipid, 54.5\%; carbohydrate, 28.3\% (sucrose, $16.6 \%$ ) by calories; Oriental Yeast) from 5 weeks of age. All the mice were individually housed. There were eleven MR flox/flox (control) mice aged 5-13 weeks, ten aged 14-16 weeks, nine aged 17 weeks, seven aged 18-19 weeks, six aged 20-21 weeks and five aged $>22$ weeks. There were twelve AdipoMR-KO mice aged 5-13 weeks, eleven aged 14-16 weeks, ten aged 17 weeks, eight aged 18-19 weeks, seven aged 20-21 weeks and six aged $>22$ weeks. Their body weight (BW) was monitored every week and blood glucose levels were measured every 4 weeks after 4 -h fasting ending at 13:00. After 14 weeks of HFHSD feeding, insulin $(1.8 \mathrm{IU} / \mathrm{kg} \mathrm{BW})$ was intraperitoneally injected, and blood glucose levels were measured using samples obtained from the tail vein at 0 , 15 , 30, 60, 90 and $120 \mathrm{~min}$ after injection. Furthermore, after 16 weeks of HFHSD feeding, glucose $(1 \mathrm{~g} / \mathrm{kg} \mathrm{BW})$ was intraperitoneally injected and blood glucose levels were measured in samples obtained from the tail vein at $0,15,30,60,90$ and $120 \mathrm{~min}$ after injection. Blood glucose levels were measured using Glutest Neo (Sanwa Kagaku Kenkyusho, Nagoya, Japan). After 18 weeks of HFHSD feeding, mice were fasted from 09:00 to 13:00, anesthetized using intraperitoneally injected pentobarbital $(100 \mathrm{mg} / \mathrm{kg}$ BW), and finally killed. The harvested tissues were weighed and subsequently frozen in liquid nitrogen.

\section{Isolation of adipocytes and stromal vascular fraction (SVF)}

Male MR flox/flox and AdipoMR-KO mice (28-weekold; $n=3$ for each) that were individually housed and fed normal chow were fasted between 09:00 and 13:00, anesthetized, and subsequently killed. Epididymal fat (Epi fat) was washed with PBS and then finely minced in DMEM. The extracellular matrix was digested using collagenase $(40,000 \mathrm{U} / 15 \mathrm{~mL}$ media) with DNase for over $40 \mathrm{~min}$ at $37^{\circ} \mathrm{C}$ in a water bath. The digested tissues were then filtered through a $70-\mu \mathrm{m}$ nylon filter and mixed with DMEM to a final volume of $50 \mathrm{~mL}$. The samples were then centrifuged at $500 \mathrm{~g}$ for $5 \mathrm{~min}$ at room temperature. Floating adipocytes were collected and washed three times with DMEM. After removing DMEM, TRI reagent (Sigma) was poured over the adipocytes. The same reagent was also poured over pelleted stromal vascular cells. These lysates were frozen and processed for RNA extraction with an RNeasy Mini kit (Qiagen).

\section{Primary cell culture}

Paired female MR flox/flox and AdipoMR-KO mice were individually housed and fed normal chow. The mice were killed at 14 weeks of age. Subcutaneous adipose tissues were obtained and immediately minced as previously described. The minced tissues were then strained by a $40 \mu \mathrm{m}$ nylon membrane. The filtrated cells were collected and placed on the dish with DMEM. After confluence, the cells were differentiated with IBMX $(0.5 \mathrm{mM})$, insulin $(1 \mu \mathrm{M})$, dexamethasone $(1 \mu \mathrm{M})$ and pioglitazone $(10 \mu \mathrm{M})$. On day 7 from differentiation, the cells were harvested and used for mRNA extraction. 


\section{Quantitative real-time PCR (RT-PCR)}

Frozen tissues or cells were treated with TRI reagent (Sigma), and total RNA was extracted with the RNeasy Mini kit (Qiagen). The RNA was reverse transcribed by Prime Script RT Master Mix (Takara). The sequences of primers are described in Supplementary Table 1 (see section on supplementary data given at the end of this article). Quantitative RT-PCR was performed in a Light Cycler System (Roche Diagnostics) with Thunderbird SYBR qPCR Mix (Toyobo, Osaka, Japan) according to the instructions provided by the manufacturer. The mRNA levels were expressed relative to those of cyclophilin A mRNA.

\section{Measurement of blood metabolites and steroids}

Blood samples were collected from anesthetized mice just before killing. Insulin levels were measured using a Morinaga Ultra-Sensitive Insulin ELISA kit (Morinaga, Kanagawa, Japan) according to the protocol supplied by the manufacturer. The levels of non-esterified fatty acid (NEFA), triglyceride (TG), total cholesterol (T-Chol) and high-density lipoprotein cholesterol (HDL-C) were determined by the NEFA C-test, TG E-test, Cholesterol E-test and HDL cholesterol E-test (Wako, Osaka, Japan), respectively. Plasma corticosterone levels were measured as previously reported (Bose et al. 2016). Plasma aldosterone levels were measured using Parameter Aldosterone Assay ( $R \& D$ systems) according to the procedure provided by the manufacturer. Adipocytokines levels were measured using Adiponectin ELISA kit (Otsuka Pharma, Tokyo), Morinaga Leptin Mouse/Rat ELISA kit (Morinaga), Quantikine ELISA MCP-1 (R\&D systems), and Quantikine ELISA Resistin (R\&D systems) as described in the ELISA kits.

\section{Measurement of tissue 8-isoprostane levels}

Tissue 8-isoprostane in Epi fat was extracted and measured using the 8-Isoprostane EIA Kit (Cayman Chemical) according to the protocol recommended by the manufacturer. Briefly, Epi fat was lysed, incubated with potassium hydroxide, trapped with Sep-Pak (Waters, Milford, MA), washed with water and hexane, eluted with ethyl acetate, evaporated and reconstituted with EIA buffer. Tissue 8-isoprostane levels were expressed relative to the protein concentration estimated by a BCA Protein Assay Kit (Thermo Fisher Scientific).

\section{Liver TG content}

Frozen liver tissues of HFHSD-fed mice were treated with the detergent 5\% nonidet-P40 (Nacalai tesque, Kyoto, Japan). The solution was sonicated and heated up to $90^{\circ} \mathrm{C}$ for 5 min twice. TG concentration in the supernatant was evaluated using the TG E-test, and the value was expressed relative to the protein concentration estimated by the BCA Protein Assay Kit (Thermo).

\section{Data analysis}

All the data are presented as the mean \pm standard error (s.E.M.). Differences between groups were analyzed by the Student's t-test. All $P$ values of $<0.05$ were considered significant. The statistical analyses were performed using JMP software (SAS Institute).

\section{Results}

\section{Generation of AdipoMR-KO mice}

We generated the AdipoMR-KO mice by crossing homozygous MR-floxed (McCurley et al. 2012) and Adiponectin-Cre mice. They constitutively express the Cre recombinase under the control of adiponectin promoter (Eguchi et al. 2011). In contrast to the lethal phenotype of whole-body MR knockout (Berger et al. 1998), AdipoMR-KO were born at the expected Mendelian ratio and appeared grossly normal, with no apparent different features or signs. In these mice, Cremediated recombination was specifically noted in the adipose tissue but not in other tissues, such as the liver and kidney (Supplementary Fig. 1). To assess the extent of MR deletion at the transcriptional level, we isolated $\mathrm{Mr}$ mRNA from various tissues and analyzed its expression level by qRT-PCR. The expression levels of $\mathrm{Mr}$ were significantly reduced in the adipose tissue, including brown adipose tissue (BAT), subcutaneous adipose tissue (Sub fat) and Epi fat, but not in the liver and kidney (Fig. 1A). Next, we separated the epididymal adipose tissue into mature adipose fraction (MAF) and SVF. The fractionation efficiency was confirmed by the high levels of adiponectin and dipeptidyl peptidase-4 (Dpp4) in MAF (Maeda et al. 1996) and SVF (Shin et al. 2017), respectively (Supplementary Fig. 2). Fractionation analysis showed that $M r$ expression levels were approximately 70 times lower in the MAF of KO mice than those in the control but not in SVF (Fig. 1B). 
A
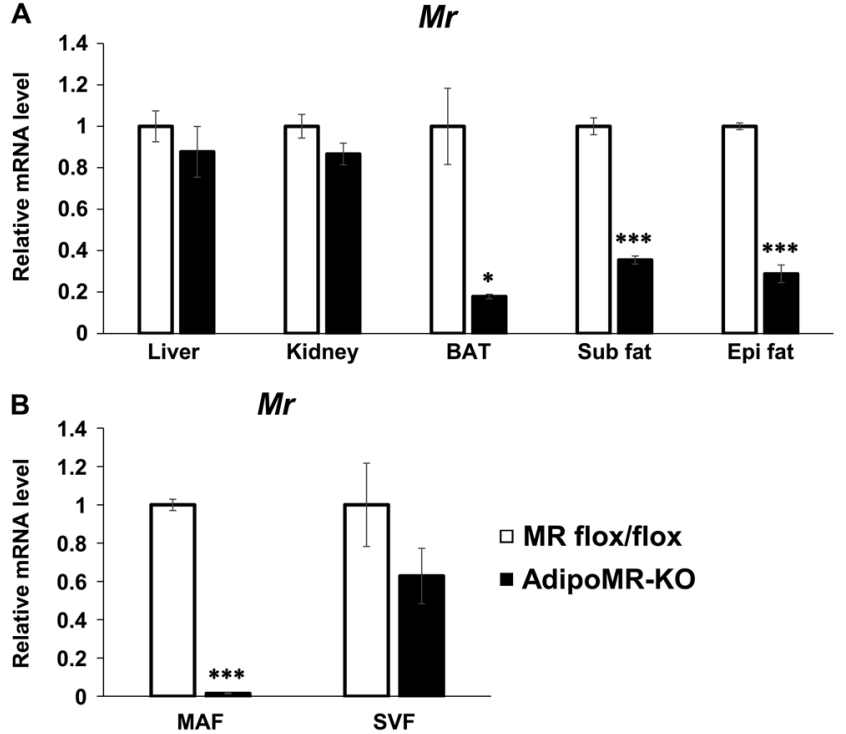

Figure 1

Generation of adipocyte-specific MR KO mice. (A) Mr mRNA expression level relative to cyclophilin A was assessed by qRT-PCR in the liver, kidney, interscapular brown adipose tissue (BAT), subcutaneous white adipose tissue (Sub fat) and epididymal fat (Epi fat) of MR flox/flox and Adipocyte-specific MR-knockout mice (AdipoMR-KO). ( $n=3$, for each). (B) $M r$ mRNA expression relative to cyclophilin $A$ was also assessed in the mature adipocyte fraction (MAF) and stromal vascular fraction (SVF) of epididymal white adipose tissue ( $n=3$, for each). Open bars, MR flox/flox mice; solid bars, AdipoMR-KO mice. Data are presented as mean \pm s.E.M. $* P<0.05, * * * P<0.001$.

\section{Effect of adipocyte MR deletion on body and organ weight}

To validate the effect of adipocyte MR deletion on obesity-induced metabolic disorders, male mice were fed HFHSD from 5 weeks of age for 18 weeks. The change in BW during the study was similar in both the AdipoMR-KO and control mice (Fig. 2A). Furthermore, there were no differences in adipose tissue weights, including Sub fat, Epi fat and BAT between the control and AdipoMR-KO mice (Fig. 2B). There were no significant differences in adipocyte size between the two groups (Supplementary Fig. 3). However, the liver weight was lower in AdipoMR-KO than in the control mice (Fig. 2B).

When mice were fed normal chow diet, BW was similar in both the AdipoMR-KO and control mice (Supplementary Fig. 4A). Furthermore, there were no differences in organ weights, including liver, kidney, mesenteric fat (Mes fat), Epi fat, Sub fat, muscle and BAT between the control and AdipoMR-KO mice (Supplementary Fig. 4B).
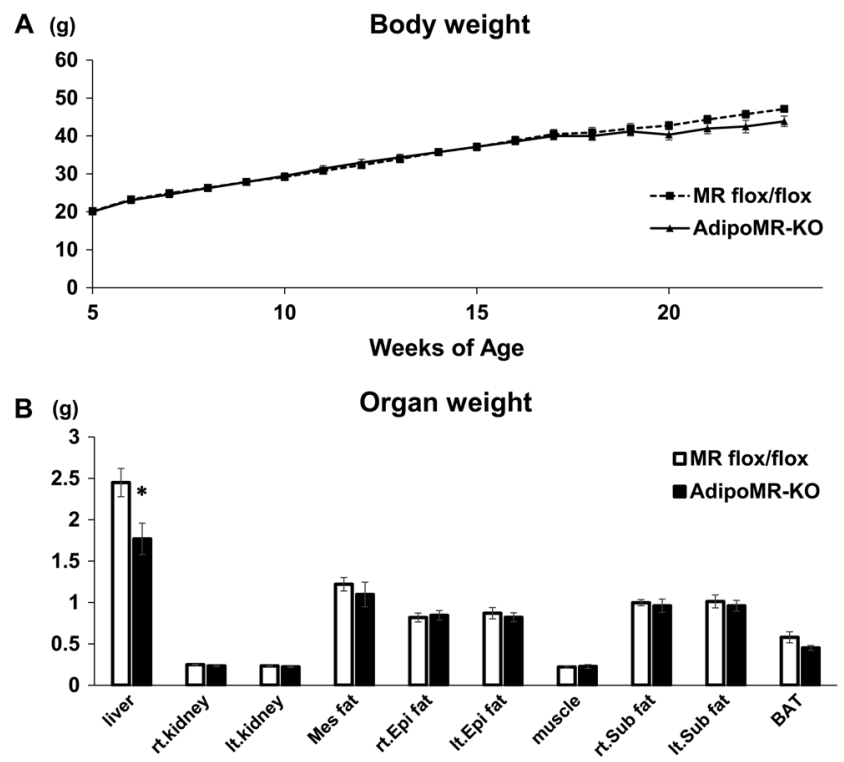

Figure 2

Effect of adipocyte MR deletion on body and organ weight. (A) Male MR flox/flox mice and AdipoMR-KO mice were fed HFHSD for 18 weeks from 5 weeks of age ( $n=11$ for mice aged $5-13$ weeks, $n=10$ for mice aged 14-16 weeks, $n=9$ for mice aged 17 weeks, $n=7$ for mice aged 18-19 weeks, $n=6$ for mice aged 20-21 weeks and $n=5$ for mice aged $>22$ weeks in the MR flox/flox group; $n=12$ for mice aged 5-13 weeks, $n=11$ for mice aged $14-16$ weeks, $n=10$ for mice aged 17 weeks, $n=8$ for mice aged 18-19 weeks, $n=7$ for mice aged 20-21 weeks and $n=6$ for mice aged $>22$ weeks in the AdipoMR-KO group). (B) Organ weights of MR flox/flox and AdipoMR-KO mice. Mice fed HFHSD from 5 weeks of age were killed at 23 weeks of age and the weights of different tissues were measured. The liver, kidney, mesenteric fat (Mes fat), epididymal fat (Epi fat), right soleus and gastrocnemius muscles (muscle), subcutaneous fat (Sub fat) and brown adipose tissue (BAT) were weighed ( $n=5$ in the MR flox/flox group; $n=6$ in the AdipoMR-KO group). Data are presented as mean \pm S.E.M. ${ }^{*} P<0.05$.

\section{Effect of adipocyte MR deletion on glucose or lipid metabolism and plasma adipocytokine levels}

To evaluate the effect of MR deletion on glucose homeostasis, blood glucose levels were measured every 4 weeks during HFHSD feeding. There were no significant differences in fasting glucose levels between the two groups (Fig. 3A). Fasting insulin levels had no significant differences between the two groups (Fig. 3B). In addition, plasma NEFA, TG, T-Chol HDL-C, aldosterone and corticosterone levels were not significantly different between the two groups (Fig. 3C, D, E, F, G and H). There were no significant differences in plasma adipocytokine levels between the two groups, including adiponectin, leptin, resistin and MCP-1 levels (Fig. 3I, J, K and L). We performed an insulin tolerance test (ITT) at 14 weeks and an intraperitoneal glucose tolerance test (GTT) at 16 weeks after HFHSD feeding. The results showed no significant 


\begin{tabular}{|l|l|l|l|l|}
\hline $\begin{array}{l}\text { Journal of } \\
\text { Endocrinology }\end{array}$ & T Hayakawa et al. & $\begin{array}{l}\text { Mineralocorticoid receptor in } \\
\text { adipocytes }\end{array}$ & $\mathbf{2 3 9 : 1}$ & $\mathbf{6 7}$ \\
\hline
\end{tabular}

A
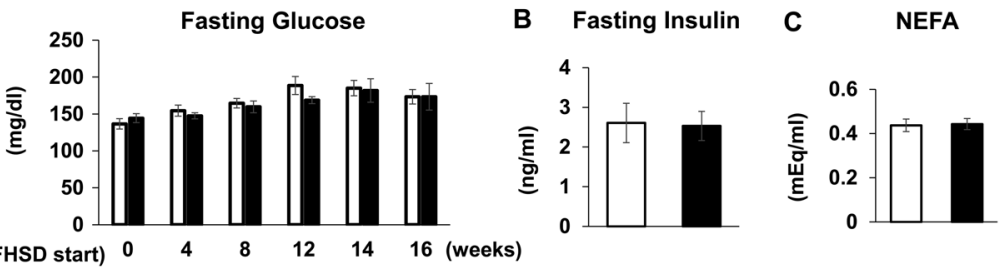

(HFHSD start) 0
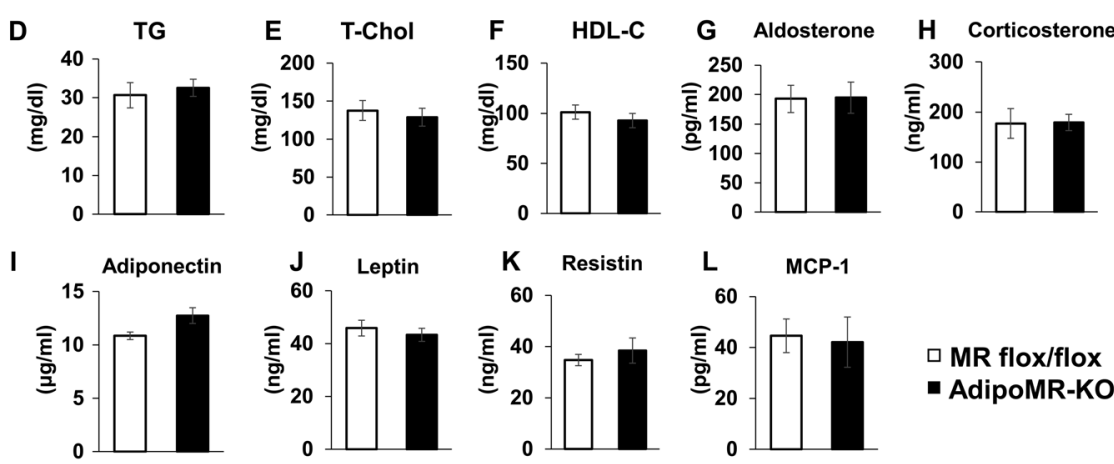

$\square$ MR flox/flox

- AdipoMR-KO

differences between the control and AdipoMR-KO mice (Fig. 4A and B). Collectively, deletion of adipose MR did not affect glucose or lipid metabolism and plasma adipocytokine levels. When mice were fed a normal chow diet, fasting plasma glucose and insulin levels were not significantly different between the two groups (Supplementary Fig. 4C and D).

\section{Effect of adipocyte MR deletion on fat mRNA and 8-isoprostane}

We analyzed the effects of MR deficiency on Epi fat mRNA (Fig. 5 and Supplementary Fig. 5). Regarding other steroid receptors (Fig. 5A), there were no significant differences in the expression levels of glucocorticoid receptor (Gr), estrogen receptor (Er) alpha and $11 \mathrm{bHSD} 1$ between the control and AdipoMR-KO mice. We also measured the mRNA levels of lipogenic and lipolytic enzymes and found no significant differences in Acaca, Fasn, Scd1, Acly, HSL, Atgl and Angptl4 between the two groups (Fig. 5B and C). We also validated the differentiation marker for adipocytes (Fig. 5D). No changes were observed in the mRNA levels of adiponectin, leptin and PPAR $\gamma$. Regarding the inflammation-related genes, the mRNA levels of $I L-6$ and lipocalin 2 did not differ, whereas those of PAI1 tended to be lower in AdipoMR-KO mice (Fig. 5E). Furthermore, there were no differences in the oxidative stress marker; 8-isoprostane (Fig. 5F), and the expression levels of related genes, such as Cyba, Sod1 and catalase (Fig. 5G). The mRNA levels of thermogenesis genes, such as Ucp1 and Ppargc1a, were not different in Epi fat

between the two groups (Fig. 5H). In BAT, the mRNA levels of Ucp1were not different between the two groups (Fig. 5I).

Next, we measured mRNA levels of primary differentiated adipocytes from Sub fat of MR flox/flox and AdipoMR-KO mice. Both preadipocytes differentiated
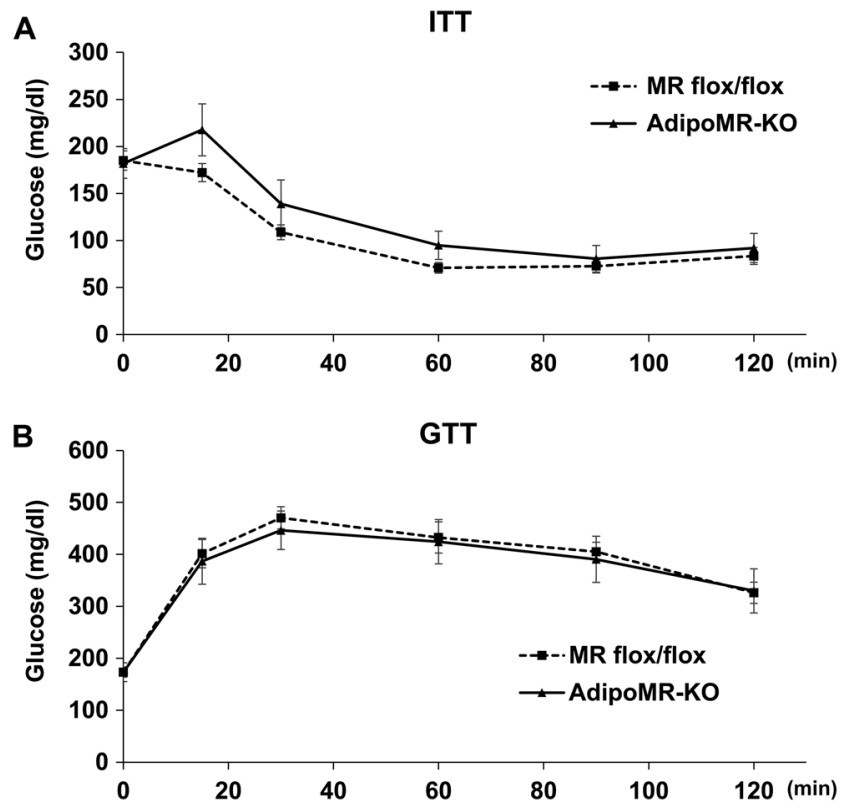

Figure 4

Effect of adipocyte MR deletion on insulin sensitivity and glucose handling. Mice fed HFHSD from 5 weeks of age underwent insulin tolerance test (ITT) 14 weeks after HFHSD feeding (A, $n=7$ in the MR flox/flox group; $n=8$ in the AdipoMR-KO group) and intra-peritoneal glucose tolerance test (GTT) 16 weeks after HFHSD feeding (B, $n=6$ in the MR flox/flox group; $n=7$ in the AdipoMR-KO group). Data are presented as mean \pm S.E.M.

\section{Figure 3}

lipid metabolism and plasma adipocytokine or steroid levels. HFHSD-fed male mice were fasted after $4 \mathrm{~h}$. (A) Fasting glucose levels $0,4,8,12,14$, weeks after HFHSD feeding $(n=11$ for 0,4 and and $n=6$ for 16 weeks in the MR flox/flox group $n=8$ for 14 weeks, and $n=7$ for 16 weeks in the AdipoMR-KO group). (B) Blood insulin levels 12 weeks after HFHSD feeding ( $n=7$ in the MR
flox/flox group; $n=8$ in the AdipoMR-KO group). (C) Plasma NEFA, (D) TG, (E) T-Chol (F) HDL-C, (G) (J) leptin, (K) resistin and (L) MCP-1 levels in blood samples collected close to sacrifice $(n=5$ in the MR flox/flox group; $n=6$ in the AdipoMR-KO AdipoMR-KO mice. Data are mean \pm s.E.M. 
A

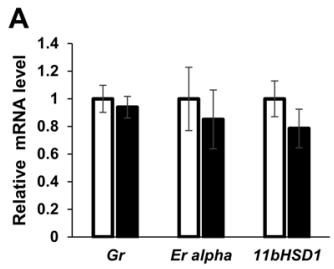

D

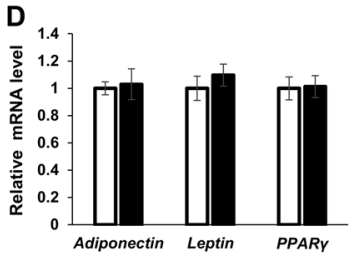

$\mathbf{G}_{1.2}$

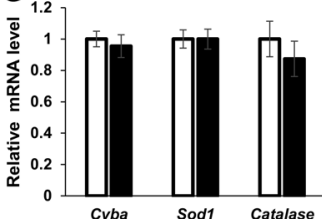

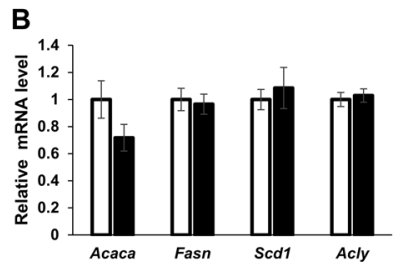
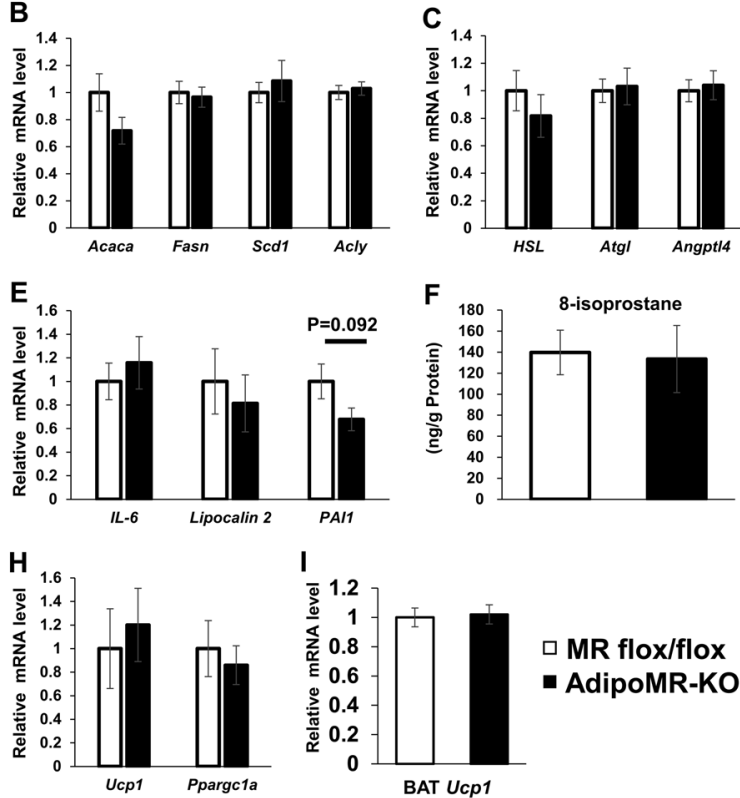

Figure 5

Epididymal fat mRNA analysis and 8-isoprostane levels, and BAT Ucp1 levels in HFHSD model.

(A) Epididymal fat mRNA levels of steroid receptor and steroid metabolic enzyme genes. (B) Lipogenic genes. (C) Lipolytic genes. (D) Adipocytokine genes and (E) other secretary cytokine genes. (F) 8-Isoprostane, a marker of oxidative stress in epididymal fat. (G) Epididymal fat mRNA levels of oxidative stress-associated genes. (H) Thermogenesis genes. (I) BAT Ucp1 mRNA levels. ( $n=5$ in the MR flox/flox group; $n=6$ in the AdipoMR-KO group.) Open bars, MR flox/flox mice; solid bars, AdipoMR-KO mice. Data are presented as mean \pm S.E.M. into mature adipocytes with the accumulation of lipid droplets (Fig. 6A). We confirmed a lower expression of $\mathrm{Mr}$ in mature adipocytes from $\mathrm{KO}$ mice (Fig. 6B). In other genes, except lipocalin 2, there were no significant differences between the two groups (Fig. 6B, C, D, E and F). Expression levels of lipocalin 2 were significantly reduced in primary differentiated adipocytes of AdipoMR-KO mice compared with those of the MR flox/flox mice (Fig. 6F), which is consistent with a previous report (Urbanet et al. 2015).

\section{Effect of adipocyte MR deletion on liver mRNA and TG content}

Finally, we analyzed liver mRNA levels on lipogenesis (Fig. 7A). The levels of lipogenic genes were not significantly different between the two groups, whereas liver TG content tended to be lower in the AdipoMR-KO mice than the control mice (Fig. 7B). Furthermore, analysis of the mRNA levels of gluconeogenesis genes showed no effect of adipocyte MR deletion on G6Pase and PEPCK mRNA levels (Fig. 7C).

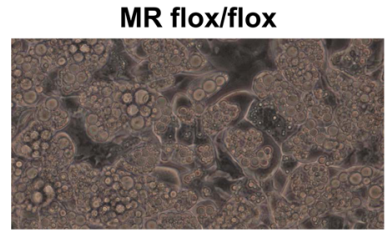

AdipoMR-KO

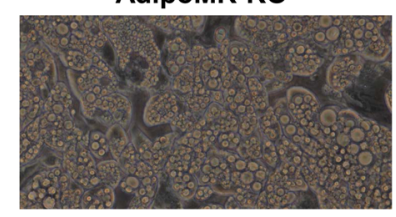

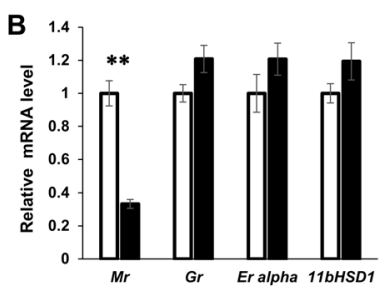

E

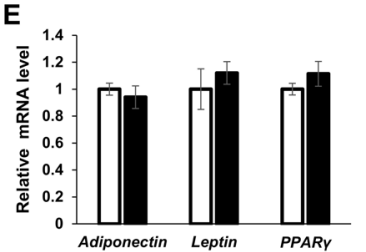

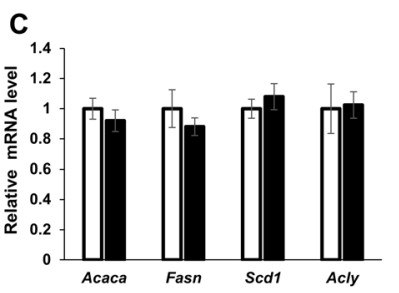

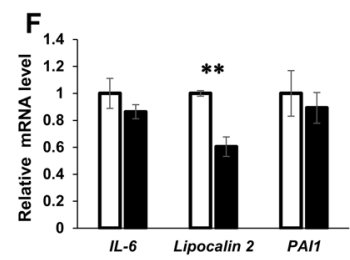

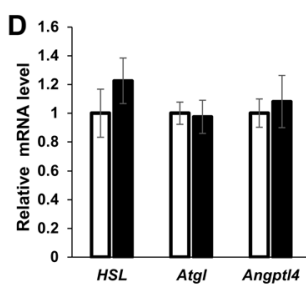

$\square$ MR flox/flox - AdipoMR-KO http://joe.endocrinology-journals.org https://doi.org/10.1530/JOE-18-0026
(C) 2018 Society for Endocrinology Published by Bioscientifica Ltd. Printed in Great Britain
Figure 6

mRNA levels and appearance of mature primary adipocytes from MR flox/flox and AdipoMR-KO mice. (A) Magnification image (200x) of mature primary adipocytes on day 7 from MR flox/flox and AdipoMR-KO mice. The levels of (B) steroid-related genes, (C) lipogenic genes, (D) lipolytic genes, (E) adipocytokine genes and (F) inflammation genes. Open bars, MR flox/flox mice; solid bars, AdipoMR-KO mice. Data are presented as mean \pm s.E.M. ${ }^{*} P<0.01$. A full colour version of this figure is available at https://doi. org/10.1530/JOE-18-0026. 
A
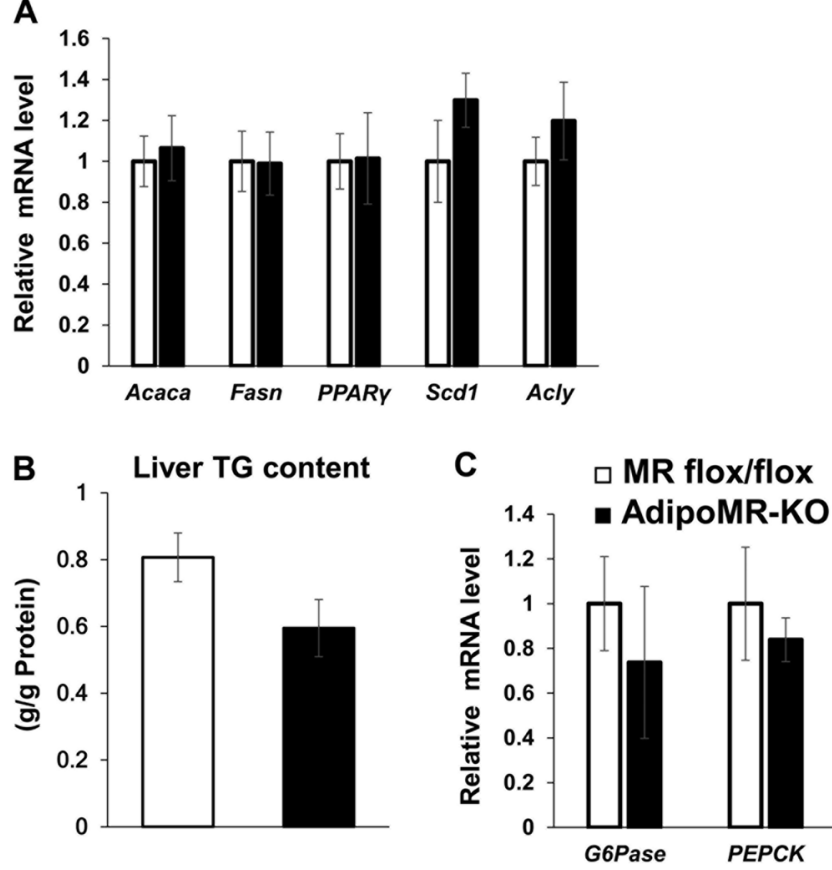

Figure 7

Liver mRNA levels and TG content in MR flox/flox and AdipoMR-KO mice of the HFHSD model. (A) mRNA expression levels of lipogenic genes. (B) Liver TG content, as measured after HFHSD feeding. (C) Gluconeogenesis-related genes (G6Pase and PEPCK). ( $n=5$ in the MR flox/flox group; $n=6$ in the AdipoMR-KO group.) Open bars, MR flox/flox mice; solid bars, AdipoMR-KO mice. Data are presented as mean \pm S.E.M.

\section{Discussion}

In obesity, active glucocorticoid levels are locally increased in adipose tissues (Masuzaki et al. 2001; Engeli et al. 2004; Patsouris et al. 2009). Adipocytes, immune cells, as well as other cells are considered to be possible target cells of glucocorticoid, which can bind and activate both MR and GR (Reul \& de Kloet 1985). There is no consensus on the target cells and molecules for excess glucocorticoid in the adipose tissue of obesity. In the present study, we generated novel AdipoMR-KO mice and showed that adipose MR has a minor role in insulin resistance and inflammation. Recently, adipocyte-specific GR-deficient mice models were generated by three different groups (Desarzens \& Faresse 2016; Mueller et al. 2017; Shen et al. 2017). Similar to AdipoMR-KO mice, these adipocyte-specific GR-deficient mice showed no significant differences in BW gain and adipose tissue formation compared with controls (Desarzens \& Faresse 2016; Shen et al. 2017). Moreover, GR deficiency worsened diet-induced inflammation, as demonstrated by higher expression levels of proinflammatory genes and macrophage infiltration in the fat pads (Desarzens \& Faresse 2016). In sharp contrast to adipocyte-specific MR-KO and GR-KO mice, obese myeloid-specific MR-KO mice exhibited improved glucose intolerance, insulin resistance and hepatic steatosis (Zhang et al. 2017). Additionally, Wada et al. (Wada et al. 2017) reported recently that aldosterone induces ROS generation in bone marrow-derived macrophages, and that eplerenone, a specific MR antagonist, inhibits the activation of NLRP3 inflammasome and ROS generation. Considering that eplerenone ameliorated inflammation and insulin resistance in ob/ob mice with suppression of macrophage infiltration and ROS production in the adipose tissues (Guo et al. 2008; Hirata et al. 2009), it is possible that MR in macrophage may play a role mainly in inflammation and insulin resistance induced by locally activated glucocorticoid in adipose tissues.

Our data showed significant reduction in liver weight of AdipoMR-KO mice in the HFHSD model. The liver TG content also tended to decrease, whereas the expression levels of lipogenic genes were not affected in AdipoMR-KO mice. The data were partially consistent with those of previous studies. In high-fat diet-induced obese animals, treatment with eplerenone induced significant reduction in liver mass and tended to reduce liver TG content (Wada et al. 2017). Another study reported that mice of ob/ob background with myeloid MR knockout had decreased liver mass and TG content without changes in Fasn and Acaca mRNA levels (Zhang et al. 2017). $\mathrm{Ob} / \mathrm{ob}$ mice spontaneously develop more severe forms of obesity, insulin resistance and steatosis than those by the HFHSD model of C57BL/6 background. Therefore, it may be difficult to detect significant differences in liver TG content between the control and AdipoMR-KO in HFHSD models.

The influx of NEFA into the liver through lipolysis of adipose tissue leads to liver steatosis. In the present study, there were no significant changes in plasma NEFA levels or the expression levels of lipolytic genes in the adipose tissue. In addition to lipolytic gene expression, lipolysis is stimulated by catecholamines that act via the $\beta$-adrenergic receptors, followed by activation of cAMPdependent protein kinase (PKA), ATGL and HSL, and their recruitment to the lipid droplet-associated surface proteins (Duncan et al. 2007). Christ et al. reported the effect of MR to enhance $\beta$-adrenergic cAMP signaling in a renal cell line (Christ et al. 2005). Precise mechanisms are unknown, however, such non-genomic effects might account for the reduction of liver weight in AdipoMR-KO mice. 
Our results showed that adipocyte-specific MR deletion in the HFHSD model was not associated with overall significant changes. This finding may be related to the expression level of $M r . M r$ expression in murine adipose tissue was approximately $2 / 3$ of that in the kidney (Supplementary Fig. 6A). In addition, fractionation analysis indicated that the mRNA level of $\mathrm{Mr}$ in MAF was only $25 \%$ of that in SVF, including immune cells, endothelial cells and preadipocytes (Supplementary Fig. 6B). Furthermore, $M r$ expression further decreased by approximately $40 \%$ after differentiation of 3T3-L1 cells into mature adipocytes (Supplementary Fig. 6C). Taken together, it seems that $\mathrm{Mr}$ expression is relatively low in adipocytes. Interestingly, glucocorticoid and aldosterone can activate MR in 3T3-L1 cells; aldosterone was reported to downregulate the expressions of adiponectin and anti-oxidative enzymes but upregulate those of inflammatory cytokines and ROS-generating enzymes in a MR-dependent manner (Guo et al. 2008; Hirata et al. 2009). In 3T3-L1 cells, downregulation of MR markedly inhibits differentiation into mature adipocytes (Caprio et al. 2007). MR has a high affinity for glucocorticoids and occupied with basal endogenous glucocorticoid levels (Joels \& de Kloet 2017), and corticosterone concentration in adipose tissue of obese mice is almost twice that of lean mice (Patsouris et al. 2009). Contrary to expectation, the results of the present study showed no significant differences in the expression levels of adipocytokines, ROS-related enzymes and tissue 8-isoprostane between the control and AdipoMR-KO mice. Considered together, minor effect of adipose MR deletion in the current study should be attributed to its low expression in adipocytes. Nevertheless, adipose MR may still play important roles in other conditions, such as excessive glucocorticoidor aldosterone-induced metabolic syndrome models or caloric restriction models.

In the current study, we used adiponectin-Cre mice to delete $\mathrm{Mr}$ specifically in adipocytes. Adiponectin-Cre is active in fully mature adipocytes in adult animals (Eguchi et al. 2011); therefore, we could not detect the effect of $\mathrm{Mr}$ during differentiation of adipocytes. As described earlier, downregulation of $\mathrm{Mr}$ markedly inhibits the differentiation of preadipocytes into mature adipocytes (Caprio et al. 2007). Armani et al. reported that MR antagonists induce browning of white adipose tissue (Armani et al. 2014). It is controversial whether beige adipocytes are derived from the differentiation of precursor cells (Wu et al. 2012) or transdifferentiation of mature adipocytes (Cinti 2009). Taken together with our results, the effects of MR antagonist on the induction of browning in white adipose tissues may be attributed to its precursor cells but not mature adipocytes.

In summary, MR in mature adipocytes plays a minor role in controlling insulin resistance and inflammation in high-fat/high-sucrose diet-induced obese mice. Further studies are needed to understand the exact MR function(s) in adipocytes.

\section{Supplementary data}

This is linked to the online version of the paper at https://doi.org/10.1530/ JOE-18-0026.

\section{Declaration of interest}

Dr Atsunori Fukuhara belongs to endowed department of the Takeda Pharmaceutical Company, Sanwa Kagaku Kenkyusho Co., Ltd., Rohto Pharmaceutical Co., Ltd., FUJ OIL HOLDINGS INC., and Roche DC Japan. Dr Jihoon Shin belongs to endowed department of the AstraZeneca K.K., Boehringer Ingelheim, Mitsubishi Tanabe Pharma Co., MSD, Novo Nordisk Pharma, Ono Pharmaceutical Co., and Taisho Toyama Pharmaceutical Co. The funders had no role in study design, data collection and analysis, decision to publish or preparation of the manuscript.

\section{Funding}

This work was supported in part by grants from Sanofi-Aventis, Inc., Tanabe Mitsubishi Pharma Ltd., Astellas Pharma Inc., Teijin Pharma Ltd. and Pfizer, Inc.

\section{Author contribution statement}

$\mathrm{T} \mathrm{H}$ designed and performed the experiments and acquired data with the help of T O, T M, Y O, J S. A F, M O and I S designed the study, interpreted the data and wrote the manuscript. All authors reviewed the manuscript and provided final approval of the version to be published. A F is the guarantor of this work and, as such, had full access to all the data in the study and takes responsibility for the integrity of the data and accuracy of the analysis.

\section{Acknowledgments}

The authors thank Dr Pierre Chambon and Dr Iris Z Jaffe for the generous gift of the MR floxed mice, and Dr E Rosen and Dr J Eguchi for providing Adiponectin-Cre mice. They also thank Haruyo Sakamoto for the excellent technical help.

\section{References}

Armani A, Cinti F, Marzolla V, Morgan J, Cranston GA, Antelmi A, Carpinelli G, Canese R, Pagotto U, Quarta C, et al. 2014 Mineralocorticoid receptor antagonism induces browning of white adipose tissue through impairment of autophagy and prevents adipocyte dysfunction in high-fat-diet-fed mice. FASEB Journal $\mathbf{2 8}$ 3745-3757. (https://doi.org/10.1096/fj.13-245415) 
Armani A, Marzolla V, Fabbri A \& Caprio M 2015 Cellular mechanisms of MR regulation of adipose tissue physiology and pathophysiology. Journal of Molecular Endocrinology 55 R1-R10. (https://doi.org/10.1530/ JME-15-0122)

Berger S, Bleich M, Schmid W, Cole TJ, Peters J, Watanabe H, Kriz W, Warth R, Greger R \& Schutz G 1998 Mineralocorticoid receptor knockout mice: pathophysiology of $\mathrm{Na}+$ metabolism. PNAS 95 9424-9429. (https://doi.org/10.1073/pnas.95.16.9424)

Bose SK, Hutson I \& Harris CA 2016 Hepatic glucocorticoid receptor plays a greater role than adipose GR in metabolic syndrome despite renal compensation. Endocrinology 157 4943-4960. (https://doi. org/10.1210/en.2016-1615)

Caprio M, Feve B, Claes A, Viengchareun S, Lombes M \& Zennaro MC 2007 Pivotal role of the mineralocorticoid receptor in corticosteroidinduced adipogenesis. FASEB Journal 21 2185-2194. (https://doi. org/10.1096/fj.06-7970com)

Christ M, Wehling M, Kirsch E, Viengchareun S, Zennaro MC \& Lombes M 2005 Enhancement of beta-adrenergic cAMP-signaling by the mineralocorticoid receptor. Molecular and Cellular Endocrinology 231 23-31. (https://doi.org/10.1016/j.mce.2004.12.004)

Cinti S 2009 Transdifferentiation properties of adipocytes in the adipose organ. American Journal of Physiology: Endocrinology and Metabolism 297 E977-E986. (https://doi.org/10.1152/ ajpendo.00183.2009)

Desarzens S \& Faresse N 2016 Adipocyte glucocorticoid receptor has a minor contribution in adipose tissue growth. Journal of Endocrinology 230 1-11. (https://doi.org/10.1530/JOE-16-0121)

Duncan RE, Ahmadian M, Jaworski K, Sarkadi-Nagy E \& Sul HS 2007 Regulation of lipolysis in adipocytes. Annual Review of Nutrition 27 79-101. (https://doi.org/10.1146/annurev.nutr.27.061406.093734)

Eguchi J, Wang X, Yu S, Kershaw EE, Chiu PC, Dushay J, Estall JL, Klein U, Maratos-Flier E \& Rosen ED 2011 Transcriptional control of adipose lipid handling by IRF4. Cell Metabolism 13 249-259. (https:// doi.org/10.1016/j.cmet.2011.02.005)

Engeli S, Bohnke J, Feldpausch M, Gorzelniak K, Heintze U, Janke J, Luft FC \& Sharma AM 2004 Regulation of 11beta-HSD genes in human adipose tissue: influence of central obesity and weight loss. Obesity Research 12 9-17. (https://doi.org/10.1038/oby.2004.3)

Funder JW 2000 Aldosterone and mineralocorticoid receptors: orphan questions. Kidney International 57 1358-1363. (https://doi. org/10.1046/j.1523-1755.2000.00975.x)

Funder JW 2009 Reconsidering the roles of the mineralocorticoid receptor. Hypertension 53 286-290. (https://doi.org/10.1161/ HYPERTENSIONAHA.108.119966)

Guo C, Ricchiuti V, Lian BQ, Yao TM, Coutinho P, Romero JR, Li J, Williams GH \& Adler GK 2008 Mineralocorticoid receptor blockade reverses obesity-related changes in expression of adiponectin, peroxisome proliferator-activated receptor-gamma, and proinflammatory adipokines. Circulation 117 2253-2261. (https://doi. org/10.1161/CIRCULATIONAHA.107.748640)

Hirata A, Maeda N, Hiuge A, Hibuse T, Fujita K, Okada T, Kihara S, Funahashi T \& Shimomura I 2009 Blockade of mineralocorticoid receptor reverses adipocyte dysfunction and insulin resistance in obese mice. Cardiovascular Research 84 164-172. (https://doi. org/10.1093/cvr/cvp191)

Hirata A, Maeda N, Nakatsuji H, Hiuge-Shimizu A, Okada T, Funahashi T \& Shimomura I 2012 Contribution of glucocorticoidmineralocorticoid receptor pathway on the obesity-related adipocyte dysfunction. Biochemical and Biophysical Research Communications 419 182-187. (https://doi.org/10.1016/j.bbrc.2012.01.139)
Joels M \& de Kloet ER 201730 YEARS OF THE MINERALOCORTICOID RECEPTOR: The brain mineralocorticoid receptor: a saga in three episodes. Journal of Endocrinology 234 T49-T66. (https://doi. org/10.1530/JOE-16-0660)

Maeda K, Okubo K, Shimomura I, Funahashi T, Matsuzawa Y \& Matsubara K 1996 cDNA cloning and expression of a novel adipose specific collagen-like factor, apM1 (AdiPose Most abundant Gene transcript 1). Biochemical and Biophysical Research Communications 221 286-289. (https://doi.org/10.1006/bbrc.1996.0587)

Masuzaki H, Paterson J, Shinyama H, Morton NM, Mullins JJ, Seckl JR \& Flier JS 2001 A transgenic model of visceral obesity and the metabolic syndrome. Science 294 2166-2170. (https://doi.org/10.1126/ science.1066285)

McCurley A, Pires PW, Bender SB, Aronovitz M, Zhao MJ, Metzger D, Chambon P, Hill MA, Dorrance AM, Mendelsohn ME, et al. 2012 Direct regulation of blood pressure by smooth muscle cell mineralocorticoid receptors. Nature Medicine 18 1429-1433. (https:// doi.org/10.1038/nm.2891)

Mueller KM, Hartmann K, Kaltenecker D, Vettorazzi S, Bauer M, Mauser L, Amann S, Jall S, Fischer K, Esterbauer H, et al. 2017 Adipocyte glucocorticoid receptor deficiency attenuates aging- and HFD-induced obesity and impairs the feeding-fasting transition. Diabetes 66 272-286. (https://doi.org/10.2337/db16-0381)

Patsouris D, Neels JG, Fan W, Li PP, Nguyen MT \& Olefsky JM 2009 Glucocorticoids and thiazolidinediones interfere with adipocytemediated macrophage chemotaxis and recruitment. Journal of Biological Chemistry 284 31223-31235. (https://doi.org/10.1074/jbc.M109.041665)

Reul JM \& de Kloet ER 1985 Two receptor systems for corticosterone in rat brain: microdistribution and differential occupation. Endocrinology 117 2505-2511. (https://doi.org/10.1210/endo-117-6-2505)

Shen ZX, Chen XQ, Sun XN, Sun JY, Zhang WC, Zheng XJ, Zhang YY, Shi HJ, Zhang JW, Li C, et al. 2017 Mineralocorticoid receptor deficiency in macrophages inhibits atherosclerosis by affecting foam cell formation and efferocytosis. Journal of Biological Chemistry 292 925-935. (https://doi.org/10.1074/jbc.M116.739243)

Shin J, Fukuhara A, Onodera T, Yokoyama C, Otsuki M \& Shimomura I 2017 Regulation of dipeptidyl peptidase-4, its substrate chemokines, and their receptors in adipose tissue of ob/ob mice. Hormone and Metabolic Research 49 380-387. (https://doi. org/10.1055/s-0043-100115)

Urbanet R, Nguyen Dinh Cat A, Feraco A, Venteclef N, El Mogrhabi S, Sierra-Ramos C, Alvarez de la Rosa D, Adler GK, Quilliot D, Rossignol P, et al. 2015 Adipocyte mineralocorticoid receptor activation leads to metabolic syndrome and induction of prostaglandin D2 synthase. Hypertension 66 149-157. (https://doi. org/10.1161/HYPERTENSIONAHA.114.04981)

Wada T, Ishikawa A, Watanabe E, Nakamura Y, Aruga Y, Hasegawa H, Onogi Y, Honda H, Nagai Y, Takatsu K, et al. 2017 Eplerenone prevented obesity-induced inflammasome activation and glucose intolerance. Journal of Endocrinology 235 179-191. (https://doi. org/10.1530/JOE-17-0351)

Wu J, Bostrom P, Sparks LM, Ye L, Choi JH, Giang AH, Khandekar M, Virtanen KA, Nuutila P, Schaart G, et al. 2012 Beige adipocytes are a distinct type of thermogenic fat cell in mouse and human. Cell $\mathbf{1 5 0}$ 366-376. (https://doi.org/10.1016/j.cell.2012.05.016)

Zhang YY, Li C, Yao GF, Du LJ, Liu Y, Zheng XJ, Yan S, Sun JY, Liu Y, Liu MZ, et al. 2017 Deletion of macrophage mineralocorticoid receptor protects hepatic steatosis and insulin resistance through ERalpha/HGF/Met pathway. Diabetes 66 1535-1547 (https://doi. org/10.2337/db16-1354)

Received in final form 4 July 2018

Accepted 23 July 2018

Accepted Preprint published online 26 July 2018
(C) 2018 Society for Endocrinology Published by Bioscientifica Ltd.
Printed in Great Britain 